$7-2014$

\title{
Numerical Evaluation of Paris-Regime Crack Growth Rate Based on Plastically Dissipated Energy
}

\author{
Parag G. Nittur \\ University of Delaware
}

Anette M. Karlsson

Cleveland State University, a.karlsson@csuohio.edu

Leif A. Carlsson

Florida Atlantic University

Follow this and additional works at: https://engagedscholarship.csuohio.edu/enme_facpub

Part of the Mechanical Engineering Commons

How does access to this work benefit you? Let us know!

\section{Publisher's Statement}

NOTICE: this is the author's version of a work that was accepted for publication in Engineering Fracture Mechanics. Changes resulting from the publishing process, such as peer review, editing, corrections, structural formatting, and other quality control mechanisms may not be reflected in this document. Changes may have been made to this work since it was submitted for publication. A definitive version was subsequently published in Engineering Fracture Mechanics, 124, 155-166, (7-2014); 10.1016/j.engfracmech.2014.04.013

\section{Original Citation}

Nittur, P. G., Karlsson, A. M., and Carlsson, L. A., 2014, "Numerical Evaluation of Paris-Regime Crack Growth Rate Based on Plastically Dissipated Energy," Engineering Fracture Mechanics, 124-125, pp. 155-166 


\title{
Numerical evaluation of Paris-regime crack growth rate based on plastically dissipated energy
}

\author{
Parag G. Nittur ${ }^{\mathrm{a}}$, Anette M. Karlsson ${ }^{\text {b,*, Leif A. Carlsson }}{ }^{\mathrm{c}}$ \\ 'Department of Mechanical Engineering, University of Delaware, Newark, DE 19716, USA \\ ${ }^{\mathrm{b}}$ Fenn College of Engineering. Cleveland State University, Cleveland, OH 44115, USA \\ ${ }^{c}$ Department of Ocean and Mechanical Engineering, Florida Atlantic University, Boca Raton, FL 33431, USA
}

\section{Introduction}

Characterizing fatigue crack growth rate is an important part of safe life assessment of structural components. Obtaining the necessary data for use in life prediction tools requires detailed specimen preparation, pre-fatiguing the notch, fatigue crack growth rate measurements and interpretation of raw data, which are all costly and time consuming. There has been considerable efforts in developing methods to improve and optimize fatigue testing to limit the number of experimental tests and thereby reduce the overall cost without compromising accuracy. The avenues proposed for modeling fatigue crack growth and obtaining life prediction tools for crack propagation include among others, methods based on damage mechanics $[1,2]$, stress intensity factors [3,4] and energy criteria [5-9].

Modeling of fatigue crack extension based on the critical plastic dissipation criterion dates back to the work of Rice [5] and has since been the topic of many analytical [6,10-12], experimental [13-16] and numerical investigations [7,9,17]. The dissipated energy criteria have been shown to be versatile for both microscopic and macroscopic analyses of fatigue [18]. A comparative assessment of the dissipated energy and other fatigue criteria can be found in Ref. [19]. Experimental results [16] have shown that the plastically dissipated energy can be used to determine crack propagation rates under both constant amplitude and variable amplitude cyclic loading.

The plastically dissipated energy can be directly linked to the accumulation of plastic strain. In metals, plastic strain is due to dislocation motion which is associated with fatigue [5,20]. An energy based approach to fatigue crack growth was developed by Weertman [11], considering a uniform distribution of edge dislocations at the crack tip. Based on the work

\footnotetext{
* Corresponding author. Tel.: +1 216687 2558; fax: +1 2166879280.
}

E-mail address: a.karlsson@csuohio.edu (A.M. Karlsson). 


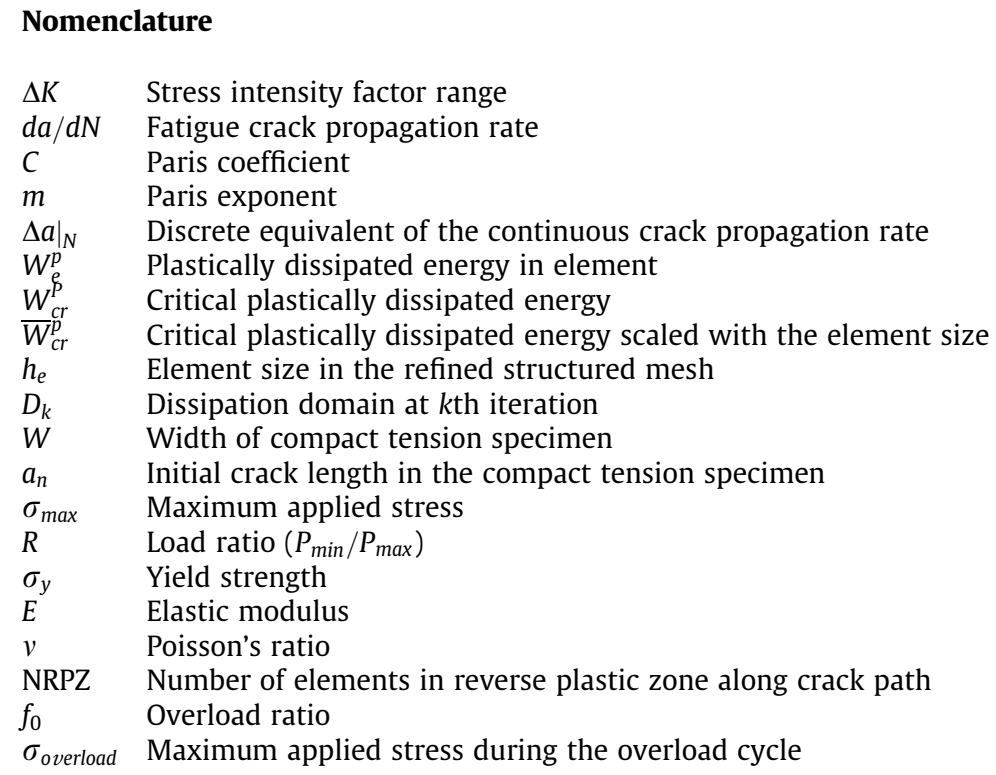

of Bodner et al. [6], Klingbeil [7] proposed a technique for predicting fatigue crack growth in terms of the per-cycle rate of plastic energy dissipated in the reverse plastic zone formed at the crack tip upon a loading-unloading cycle. Klingbeil's approach is based on evaluating the plastically dissipated energy around a stationary crack under mode I loading using finite element analysis. The technique was later applied by Daily and Klingbeil [21] for stationary cracks under mixed mode loading conditions. Klingbeil's theory was recently extended and used by Smith [17] to examine the applicability of the dissipated energy criterion for predicting delayed retardation effects following a single tensile overload. A 3D boundary layer FE model was used to model a crack arbitrarily propagating at the rate of one element per cycle. Crack tip shielding effects were also accounted for. However, the results were found to be mesh dependent due to the arbitrary crack propagation rate. Moreover, the actual crack growth rate can only be determined via experimental calibrations in that scheme [17].

An alternative approach to predict fatigue crack propagation rate directly from FE simulations based on the plastically dissipated energy criterion was recently proposed by Cojocaru and Karlsson [9]. A two dimensional (2D) plane strain analysis was presented for fatigue crack growth rate changes due to negative load ratios and single and multiple tensile overloads. Qualitative agreement with experimentally observed rates was shown for these different load cases. In addition, the plastically dissipated energy criterion was also implemented for three dimensional modeling of fatigue crack growth to predict crack front profile changes (crack tunneling) under cyclic loading [22].

When subjected to fatigue loading, materials exhibit a linear portion on a log-log plot of fatigue crack propagation rate, $d a / d N$, versus the applied stress intensity factor range, $\Delta K$. In the fracture mechanics approach to fatigue crack propagation, this linear portion of experimental data is described by the well known Paris' law [3,23]

$$
\frac{d a}{d N}=C \Delta K^{m}
$$

where $C$ and $m$ are experimentally determined material properties commonly called Paris coefficients. In this work, we augment the approach proposed in Ref. [9] for modeling crack growth under cyclic stresses based on the plastically dissipated energy to numerically determine these Paris law coefficients. Comparisons with measured crack growth rate data is presented for a variety of ductile metals, including aluminum, titanium and nickel based alloys used in aerospace applications.

\section{Numerical approach to predicting cyclic crack growth rate}

\subsection{Theory}

In this work, the numerical scheme for predicting cyclic crack propagation rate is based on a continuum perspective $[8,9]$. Our premise is that fatigue cracks propagate due to cyclic material degradation in a process zone associated with the crack tip (see for example, Ref. [24]). The degradation of the material in the process zone is accompanied by significant plastic deformation if the material is ductile (due to dislocation motion in metals, and shear banding and crazing in polymers). 
On a continuum level, dislocation motion is associated with plastic yielding. Since fatigue crack growth occur as the dislocations accumulate, it is convenient to quantify this as dissipated plastic energy [9].

Following the approach in Refs. [8,9,22], the entire cyclic path is simulated using the finite element (FE) method by conducting cycle by cycle simulations. The per-cycle crack propagation rate is not prescribed a priori, but automatically obtained by probing the plastically dissipated energy in a user-defined domain ahead of the current crack tip [8].

During the load cycle, the material ahead of the crack tip yields in tension forming a forward plastic zone (Fig. 1). During unloading, a new plastic zone of reversed deformation is formed embedded within the forward plastic zone. The material within this smaller plastic zone undergoes compressive yielding and this zone is referred to as the reverse plastic zone (Fig. 1) [5]. When the crack propagates through these yield zones, it leaves behind a wake of material that has undergone plastic deformations, commonly referred to as the plastic wake. The computational scheme is initiated by applying cyclic loading and arbitrarily propagating an initial crack every few cycles. This arbitrary propagation is continued until the crack has propagated past the initial reverse plastic zone formed at the end of first cyclic load. This technique ensures the formation of a fully developed plastic wake behind the crack tip. Once a fully developed plastic wake is formed, the discrete crack propagation rate, $\left.\Delta a\right|_{N}$ (which is the discrete equivalent of the continuous crack propagation rate $d a / d N$ ), is obtained according to the following:

At the end of cycle $N_{t}$, the dissipation domain, $D_{1}$, (Fig. 2) is defined at the tip of the current crack. This dissipation domain is associated with the crack tip and translates with the crack tip as the crack propagates. The plastically dissipated energy is evaluated in this dissipation domain. Owing to the discrete nature of the FE model, the dissipation domain is also discrete, enclosing a set of elements, $E_{D}$. The plastically dissipated energy, $W_{e}^{p}$, in an element $e$ is

$$
W_{e}^{p}=\int_{V} \sigma_{i j} d \varepsilon_{i j}^{p} d V
$$

where $V$ is the volume of the element. At the end of load cycle $N_{t}$ the plastically dissipated energy integrated over the entire domain, $W_{N_{t}}^{p}\left(D_{1}\right)$, is obtained as a summation of plastically dissipated energy over all the elements in the dissipated domain:

$$
W_{N_{t}}^{p}\left(D_{1}\right)=\left.\sum_{e \in E_{D}} W_{e}^{p}\right|_{N_{t}}
$$

After application of the next load cycle, $N_{t+1}$, the plastically dissipated energy accumulated in the dissipation domain is

$$
W_{N_{t+1}}^{p}\left(D_{1}\right)=\left.\sum_{e \in E_{D}} W_{e}^{p}\right|_{N_{t+1}}
$$

The increment in plastically dissipated energy in the dissipation domain, $\Delta W_{p}^{1}\left(D_{1}\right)$, is obtained as

$$
\Delta W_{p}^{1}\left(D_{1}\right)=W_{N_{t+1}}^{p}\left(D_{1}\right)-W_{N_{t}}^{p}\left(D_{1}\right)
$$

$\Delta W_{p}^{1}\left(D_{1}\right)$ is then compared to the critical plastically dissipated energy, $W_{c r}^{P}$ considered a material property. If $\Delta W_{p}^{1}\left(D_{1}\right)<W_{c r}^{P}$, an additional load cycle, $N_{t+2}$, is applied and the accumulated increment in the plastically dissipated energy is:

$$
\Delta W_{p}^{2}\left(D_{1}\right)=W_{N_{t+2}}^{p}\left(D_{1}\right)-W_{N_{t}}^{p}\left(D_{1}\right)
$$

Cyclic loading is continued until cycle $N_{t+i}$ at the end of which $\Delta W_{p}^{i}\left(D_{1}\right) \geqslant W_{c r}^{P}$ :

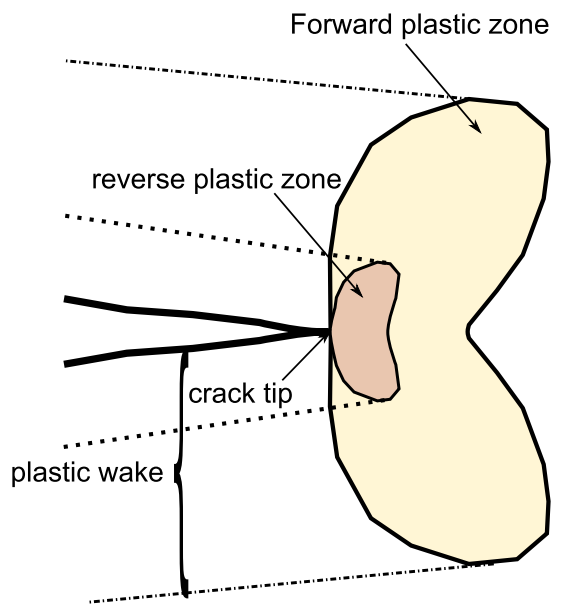

Fig. 1. Plastic regions developing during cyclic loading around a crack tip. The plastic wake develops when the crack propagates through the plastic region [9]. 


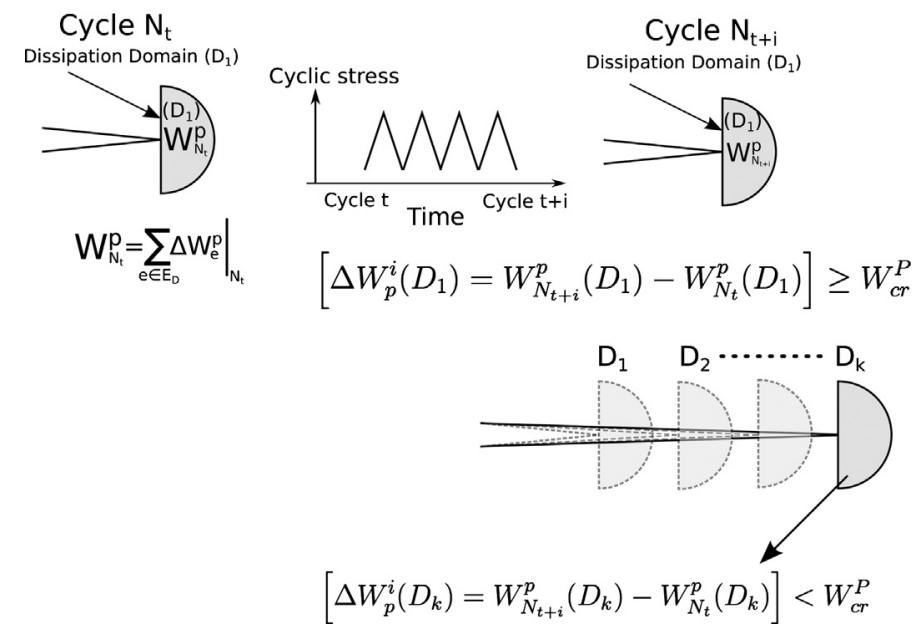

Fig. 2. Crack tip with the associated dissipation domain and illustration of the iterative scheme to obtain the discrete crack propagation rate $\left.\Delta a\right|_{N}$ (the discrete equivalent of the continuous crack propagation rate $d a / d N$ ).

$$
\left[\Delta W_{p}^{i}\left(D_{1}\right)=W_{N_{t+i}}^{p}\left(D_{1}\right)-W_{N_{t}}^{p}\left(D_{1}\right)\right] \geqslant W_{c r}^{P}
$$

When condition (7) is fulfilled, crack propagation ensues and the crack propagates by one element using a node release technique. The dissipated domain moves with the crack tip as illustrated in Fig. 2. Next, the accumulated increment in the plastically dissipated energy between cycles $N_{t}$ and $N_{t+i}$ in dissipation domain, $D_{2}$, is evaluated as:

$$
\Delta W_{p}^{i}\left(D_{2}\right)=W_{N_{t+i}}^{p}\left(D_{2}\right)-W_{N_{t}}^{p}\left(D_{2}\right)
$$

If $\Delta W_{p}^{i}\left(D_{2}\right) \geqslant W_{c r}^{P}$, the crack propagates one more element. The dissipation domain is again repositioned ahead of the new crack tip and this sequence is repeated $k$ times until

$$
\left[\Delta W_{p}^{i}\left(D_{k}\right)=W_{N_{t+i}}^{p}\left(D_{k}\right)-W_{N_{t}}^{p}\left(D_{k}\right)\right]<W_{c r}^{P}
$$

The crack will stop growing once the above criterion is satisfied. The discrete propagation rate, $\left.\Delta a\right|_{N}$, achieved between cycles $N_{t}$ and $N_{t+i}$ can be expressed as

$$
\left.\Delta a\right|_{N}=\frac{k h_{e}}{N_{t+i}-N_{t}}=\frac{k h_{e}}{i}
$$

where $h_{e}$ is the element length in the refined structured mesh.

Crack propagation rate in subsequent load cycles is evaluated using the above mentioned algorithm with $N_{t+i} \rightarrow N_{t}$ and $D_{k} \rightarrow D_{1}$ expressed in Eqs. (3) through (10). Thus, after each load cycle, the crack may be stationary or may propagate one or multiple elements.

\subsection{Implementation of cyclic analysis}

The implementation follows the framework presented in Ref. [8,9,22]. The computational scheme for cyclic crack propagation using the iterative algorithm described above is implemented in the commercially available finite element simulation package ABAQUS [25] and is divided into two main levels, Python level and the ABAQUS level, as shown in Fig. 3. The cycle by cycle simulation is automated using the ABAQUS Scripting Interface (ASI) which is an object oriented extension library based on Python [26]. ABAQUS/CAE Graphic User Interface (GUI) is used to describe the model, generate the mesh, specify loading, boundary conditions and other properties not required to be updated during the cyclic analysis. The details of the crack interface are then passed on to the Python level.

The crack interface consists of an intact portion ahead of the crack tip, i.e., the predefined crack path, and a wake portion behind the crack tip. Similar to the method used in Ref. [22], the Python ASI is used to specify "equation constraints" [25] in ABAQUS to define the intact portion of the crack interface and to update the interface at the end of each cycle as required by the crack propagation algorithm. Normal, frictionless contact formulation available in ABAQUS is prescribed at the crack wake to account for plasticity induced crack closure. The effect of roughness at the crack wake can be modeled by specifying the friction coefficient for normal contact with the friction formulation available in ABAQUS. However, for simplicity, the effect of roughness is not considered in the present work. Once the interface definitions are completed, the model is submitted to the ABAQUS solver from within the python ASI. The plastically dissipated energy within each element, $W_{e}^{p}$, is automatically 


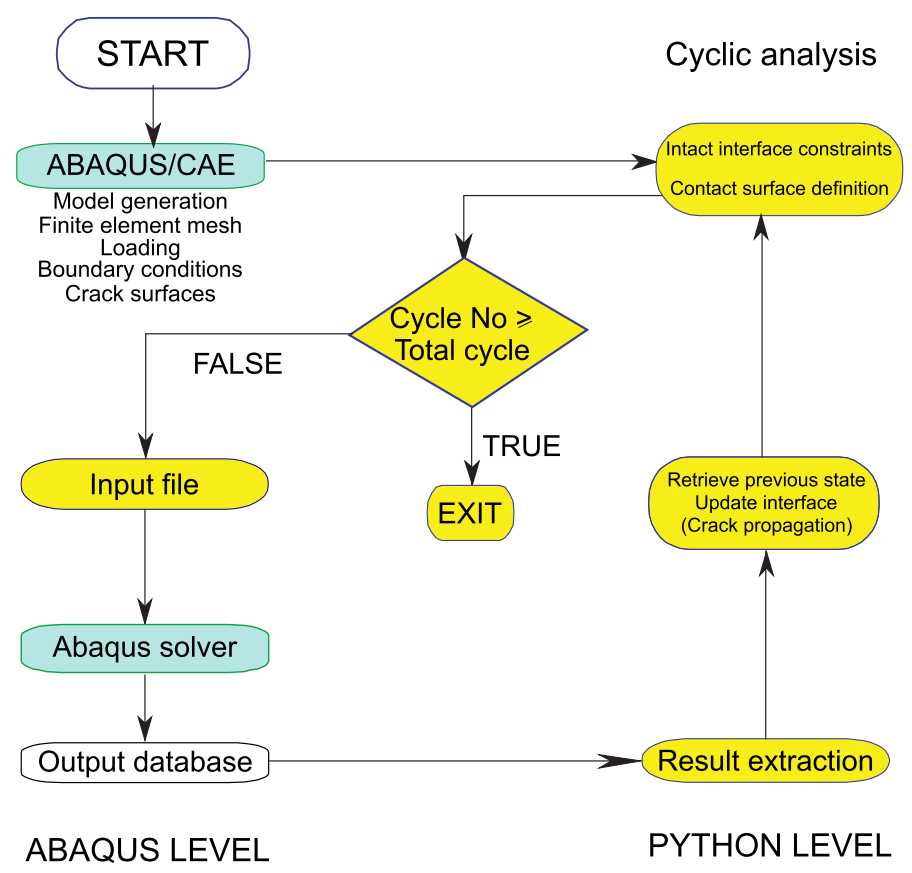

Fig. 3. Schematic of implementation in ABAQUS $[8,9,22]$ to automate cycle-by-cycle simulations and crack growth under cyclic loads.

calculated by ABAQUS during each load step as part of the solution, the output of which can be requested with the keyword 'ELPD' [25]. The output database file containing the results from the solution of the FE model is probed using the Python ASI to determine the position of the new crack tip. A node release technique is used to extend the crack and redefine the intact portion of the interface if the crack propagation criterion is fulfilled. Subsequently, contact definitions are updated to include the newly formed surface behind the crack tip. The previous converged state is imported to the interface updated model and the next cycle simulated. This whole sequence is implemented as a recursive algorithm which calls itself as many times as the number of user specified cycles.

\section{Finite element (FE) model}

\subsection{Model definition}

Cyclic crack propagation is simulated in a standard compact specimen (ASTM E 399 [27]) with a width, $W=100 \mathrm{~mm}$, and an initial crack length $a_{n}=0.2 W=20 \mathrm{~mm}$ (Fig. 4). The initial crack tip is put at the origin of the XY coordinate system. The mesh consists of standard, 4 noded, iso-parametric plane strain elements with reduced integration, 'CPE4R' [25]. Incremental small strain elastoplasticity with von Mises yield criterion is used to obtain the constitutive response of the material [25].

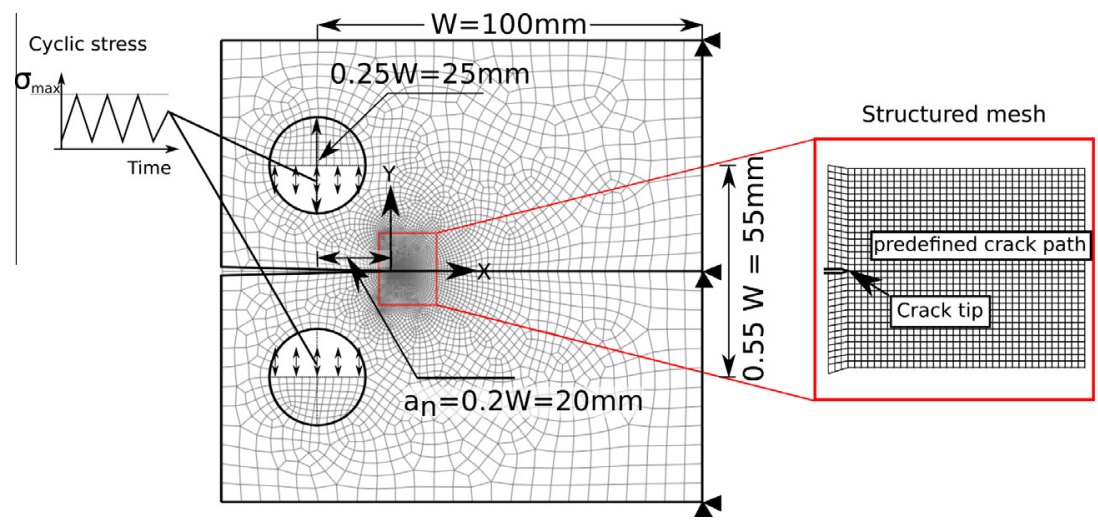

Fig. 4. Finite element model of compact specimen with loading and boundary conditions. 
Plastic dissipation occurring within each element is automatically calculated by ABAQUS during each load step. Ref. [7] investigated the effect of strain hardening on plastically dissipated energy using a bi-linear kinematic hardening model. Results from his investigations show only a weak dependence of strain hardening on the plastically dissipated energy under plane strain conditions and when the hardening modulus is within $10 \%$ of the elastic modulus as typically observed in ductile metals. Therefore, for simplicity, a linear-elastic, perfectly-plastic material behavior is assumed in all the simulations. The specimen is loaded by applying cyclic stress at the mid-plane of the loading pin holes of the compact specimen (Fig. 4). During each cycle, the stress is varied linearly from an initial minimum value of $0.1 \sigma_{\max }$ to a maximum value, $\sigma_{\max }$, so as to obtain a load ratio $R=0.1$ unless otherwise noted.

A refined structured mesh is used in a rectangular region along a predefined straight crack path as shown in the inset of Fig. 4. To model crack propagation using the scheme described in Section 2 with a node release technique, the spacing of the nodes is kept constant on both sides of the crack interface. Fatigue is a progressive process occurring during the load cycle. However, the use of discrete sized load increments and cyclic crack growth in increments of the element size discretize this physical process. There is no clear consensus on the appropriate scheme for crack advance in FE simulations of fatigue crack propagation [28]. McClung and Sehitoglu [29] examined three node release schemes: at maximum load, at minimum load and immediately after maximum load. They observed no significant differences between the three schemes with respect to crack opening load levels. Following the approach in Refs. [9,22,30-32], crack propagation is accomplished by releasing the "equation constraints" on the current crack front nodes at the end of the load cycle, i.e., at minimum load. Normal, frictionless contact formulation in ABAQUS [25] is employed at the crack wake to account for plasticity induced crack closure. The degree of mesh refinement in the region of the structured mesh is characterized by the number of elements resolving the reverse plastic zone along the crack path at the end of the first loading cycle.

\subsection{Mesh convergence of the numerical scheme}

We will first investigate mesh convergence to show that the results are independent of the mesh size. To this end, cyclic crack propagation in a standard compact specimen (Fig. 4) is simulated using the numerical scheme described in Section 2. The material properties are representative for an aluminum alloy - Al 7475-T7351 [33]. A linear-elastic, perfectly-plastic constitutive response is assumed, with yield strength $\sigma_{y}=410 \mathrm{MPa}$, elastic modulus $E=71 \mathrm{GPa}$ and Poisson's ratio, $v=0.33$ [33]. The specimen is loaded at the mid-plane of the loading pin-holes (Fig. 4) with a purely tensile, triangular, cyclic load. The load is cycled at a load ratio $R=0.1$. The applied loading corresponds to a stress intensity factor range, $\Delta K$, of $20 \mathrm{MPa} \sqrt{\mathrm{m}}$.

Fig. 5 shows the two meshes used in the convergence study, discretizing the forward and reverse plastic zones formed during the first loading cycle. For the coarse mesh, the reverse plastic zone is resolved with 2 elements along the crack path whereas the fine mesh has 4 elements. The critical plastically dissipated energy per unit area of crack extension, $W_{c r}^{p}$, is $30 \mathrm{Nmm} / \mathrm{mm}^{2}$ (see Section 3.3 for discussion on obtaining this value) for this aluminum alloy. Due to the discrete nature of finite element simulations, crack extension modeled in a homogeneous material through a node release technique is also discrete. The minimum possible crack extension thus depends on the size of the elements ahead of the crack tip. As such, there is an inherent, apparent mesh dependency. However, since the crack propagation is not arbitrary but depends on the critical plastically dissipated energy, mesh independent results are indeed obtained. The critical plastically dissipated energy is defined per unit area of crack extension and therefore can be linearly scaled with the size of the element ahead

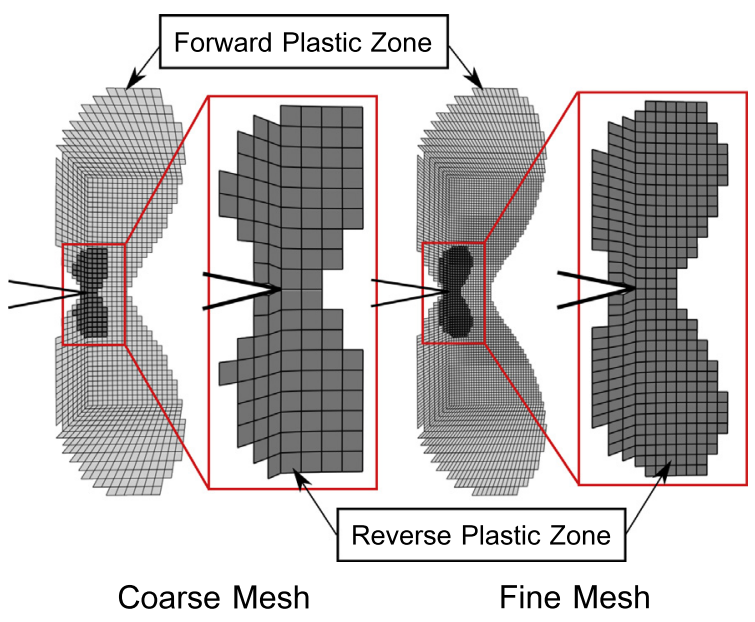

Fig. 5. Forward and reverse plastic zones after the first load cycle resolved with the coarse and the fine mesh used in the convergence study. Coarse mesh has 2 elements resolving the reverse plastic zone along the crack path whereas the fine mesh has 4. 


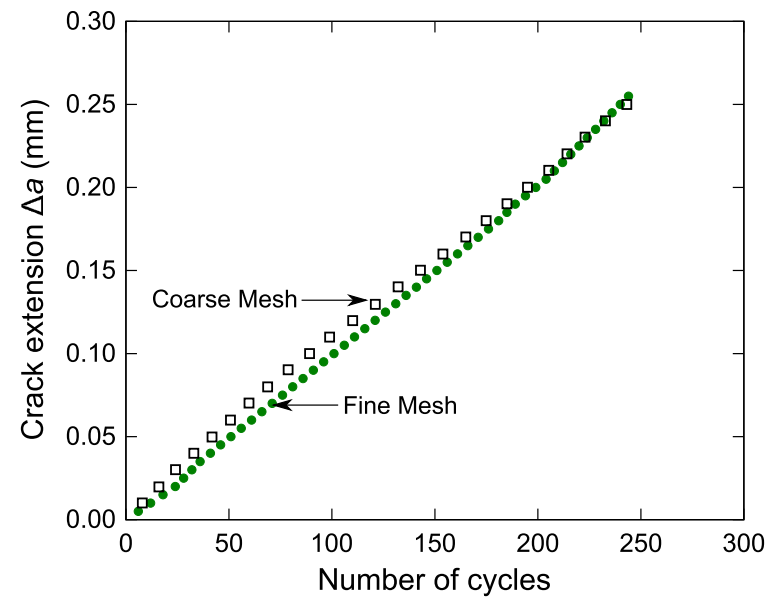

Fig. 6. Crack extension as a function of the number of cycles obtained from coarse and fine mesh simulations demonstrating mesh convergence.

of the crack tip. Element sizes of $\approx 0.01$ and $0.005 \mathrm{~mm}$ were used for the coarse and fine meshes, respectively. Scaled values of $W_{c r}^{p}, 30 \times 0.01=0.3 \mathrm{Nmm}$ and $30 \times 0.005=0.15 \mathrm{Nmm}$ are specified as input in the coarse and fine mesh simulations.

Fig. 6 shows the crack extension as a function of the number of cycles obtained from the coarse and fine mesh model simulations. The crack propagation rate $d a / d N$ is obtained from the slope of a line fitted to the data. $d a / d N=0.97$ and $1.0 \mu \mathrm{m} /$ cycle for the coarse and fine mesh models, respectively, differing by only $\approx 3 \%$ and thus confirming mesh convergence. The mesh refinement level for subsequent simulations is kept such that the reverse plastic zone is refined with at least 4 elements along the crack path. This refinement level also concurs with suggestions in literature for obtaining converged results for opening and closing loads in plasticity induced crack closure (PICC) simulations [32].

Note: The size of the dissipation domain in all the simulations is chosen so as to fully enclose the reversed plastic zone formed at the end of the first load cycle. The size of the reversed plastic zone depend on the applied loading and the load ratio. The appropriate dimensions of the dissipation domain thus depend on the applied loading. For simplicity, and to confirm with the structured mesh around the crack tip, the dissipation domain is chosen to be a rectangle as depicted in Fig. 7. The purpose of the dissipation domain is to aid in summing the increment of plastically dissipated energy in the elements that are in front of the crack tip and have undergone reverse yielding. After the first cycle, the increment in plastically dissipated energy occurs only in the reverse plastic zone. Any element that is outside the reverse plastic zone will have a zero increment in plastically dissipated energy. Therefore it is not required for the dissipation domain to confirm tightly to the reverse plastic zone. The only requirement for the size of the dissipation domain is that it fully encloses the reverse plastic zone. In the current work, only a single crack is considered but the frame work is not limited to single cracks. When multiple cracks are modeled, a dissipation domain is assigned to each crack tip [8]. The dissipation domain corresponding to each crack tip then aids in summing the increment in plastically dissipated energy ahead of the crack tip it is associated with and governs the extension of that crack tip.

\subsection{Establishing the critical plastically dissipated energy}

The critical plastically dissipated energy, $W_{c r}^{p}$, is assumed to be a material property. When the accumulated dissipated energy in the dissipation domain supersedes $W_{c r}^{p}$, the crack propagates. Thus, this parameter governs the crack propagation

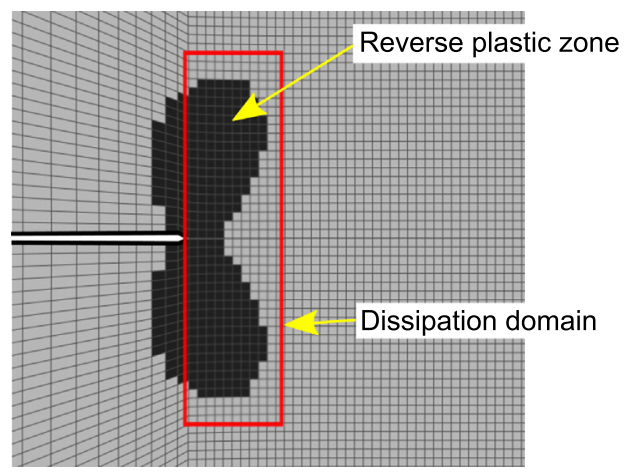

Fig. 7. The size of the rectangular dissipation domain is chosen so as to fully enclose the reverse plastic zone formed at the end of the first load cycle. 
rate. To the knowledge of the authors, there is no suitable experimental data establishing $W_{c r}^{p}$, but is an area of active ongoing research [16]. In this work, $W_{c r}^{p}$ is determined by using one set of experimentally measured data $(\Delta K, d a / d N)$ obtained from Ref. [33], according to the procedure described below.

Cyclic crack propagation in the compact specimen with loading and boundary conditions as shown in Fig. 4 is simulated. The material considered is an aluminum alloy, Al 7475-T7351, with properties mentioned in the previous section (Section 3.2). Cyclic crack growth is simulated with an applied $\Delta K$ range of $20 \mathrm{MPa} \sqrt{\mathrm{m}}$ cycled at a load ratio $R=0.1$. The crack growth rate $d a / d N$ is obtained from a linear least square fit of the crack extension versus number of cycles plot as previously described in Section 3.2. The value of $W_{c r}^{p}$ that results in the experimentally obtained $d a / d N$ is used as the critically dissipated energy. For this particular load case $(\Delta K=20 \mathrm{MPa} \sqrt{\mathrm{m}})$, the numerically evaluated $d a / d N$ matched with experimental data when $W_{c r}^{p}=0.15 \mathrm{Nmm}$. This value is then used for all load levels in the simulations of crack growth in Al 7475-T7351.

\section{Results and discussion}

In addition to the $\Delta K=20 \mathrm{MPa} \sqrt{\mathrm{m}}$ load case described above for establishing $W_{c r}^{p}$, cyclic crack propagation is simulated in the compact specimen (Fig. 4) with three more levels of applied loading ( $\Delta K=16.6,13.3$ and $10.0 \mathrm{MPa} \sqrt{\mathrm{m}}$ ) in order to determine the Paris law constants. The load ratio $R=0.1$ in all the cases.

The mesh resolution and relative sizes of the reverse plastic zone formed after the first load cycle for each of these load cases are shown in Fig. 8. Resolution of the reverse plastic zone with at least 4 elements is vital for getting reliable results from the numerical scheme. As evident in Fig. 8, the mesh size in the higher $\Delta K$ load case would inadequately resolve the reverse plastic zone at lower $\Delta K$. Using a fine mesh would enormously add unnecessary computational expense in simulating higher $\Delta K$ load cases. Consequently, different mesh sizes are used for simulating different load cases. The reverse plastic zone is discretized by four elements along the crack path in the case of $\Delta K=20 \mathrm{MPa} \sqrt{\mathrm{m}}$ load case, whereas the other load cases have 6-8 elements. The critical plastically dissipated energy $\left(30 \mathrm{Nmm} / \mathrm{mm}^{2}\right)$ is scaled according to the mesh size and specified as input in the other load cases. The mesh size and the corresponding values of $W_{c r}^{p}$ used in the simulation of four load cases are listed in Table 1.

Experimental observations, e.g., Davidson [34], Ranganathan et al. [16], suggest that the hysteresis energy dissipated per unit surface (specific energy) during crack extension is not a pure material constant. The specific energy depends on $\Delta K$ at low values of $\Delta K$ and the crack growth mechanism. The specific energy reaches a constant only at high $\Delta K$ values. Adequate resolution of the reverse plastic zone at low $\Delta K$ levels would require the use of sub-micron size elements. Therefore a high value of $\Delta K$ is selected for evaluating the critical plastically dissipated energy, $W_{c r}^{p}$.

Results for crack extension from simulated cyclic crack growth for the four levels of $\Delta K$, are presented in Fig. 9. A linear least squares fit was used to obtain the slope of the data points corresponding to each load case. The slope is the crack growth rate $d a / d N$. Measured [33] and simulated crack growth rates are summarized in Table 1 and shown in Fig. 10. The numerically calculated crack growth rates are centered within the experimental scatter band.

The applicability of this modeling approach was further examined for Inconel 718 and Titanium Ti-6Al-4V alloys. Results from the simulations are shown along with experimental data in Fig. 11. The circled data points in these plots were used for calibrating the critical plastically dissipated energy.

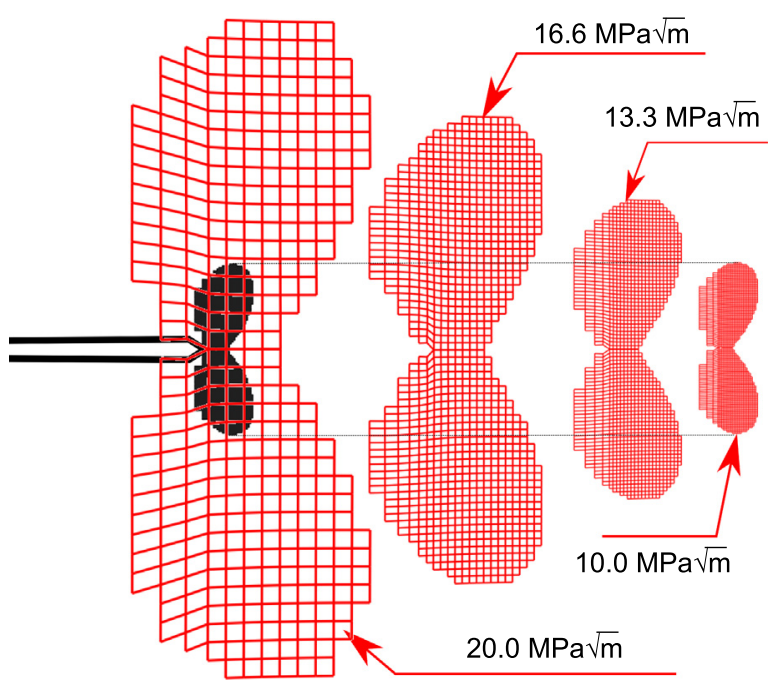

Fig. 8. Relative sizes of the reverse plastic zone formed at the end of the first load cycle for each of the load cases considered. The reverse plastic zone is discretized by four elements along the crack path in the case of $\Delta K=20 \mathrm{MPa} \sqrt{\mathrm{m}}$ load case, whereas the other load cases have $6-8$ elements. 
Table 1

Summary of crack growth results for Al 7475-T7351 from the finite element analysis. $W_{c r}^{p}=30 \mathrm{Nmm} / \mathrm{mm}^{2}$. Values of $W_{c r}^{p}$ scaled with the element size are specified as input in the simulations, e.g., $\bar{W}_{c r}^{p}=30 \times 0.005=0.15$.

\begin{tabular}{llclll}
\hline$\Delta K(\mathrm{MPa} \sqrt{\mathrm{m}})$ & Element size $(\mu \mathrm{m})$ & $\mathrm{NRPZ}^{\mathrm{d}}$ & $\bar{W}_{c r}^{p}(\mathrm{Nmm})$ & $d a / d N(\mathrm{Model})(\mu \mathrm{m} / \mathrm{cycle})$ & $d a / d N(\mathrm{Measured})(\mu \mathrm{m} / \mathrm{cycle})$ \\
\hline 20.0 & 5.0 & 4 & 0.150 & 1.34 & 1.20 \\
16.6 & 2.0 & 7 & 0.060 & 0.667 & $0.45-0.85$ \\
13.3 & 1.0 & 8 & 0.030 & 0.283 & $0.20-0.45$ \\
10.0 & 0.5 & 8 & 0.015 & 0.0833 & $0.05-0.15$ \\
\hline
\end{tabular}

${ }^{\mathrm{d}}$ Number of elements in reverse plastic zone along crack path.

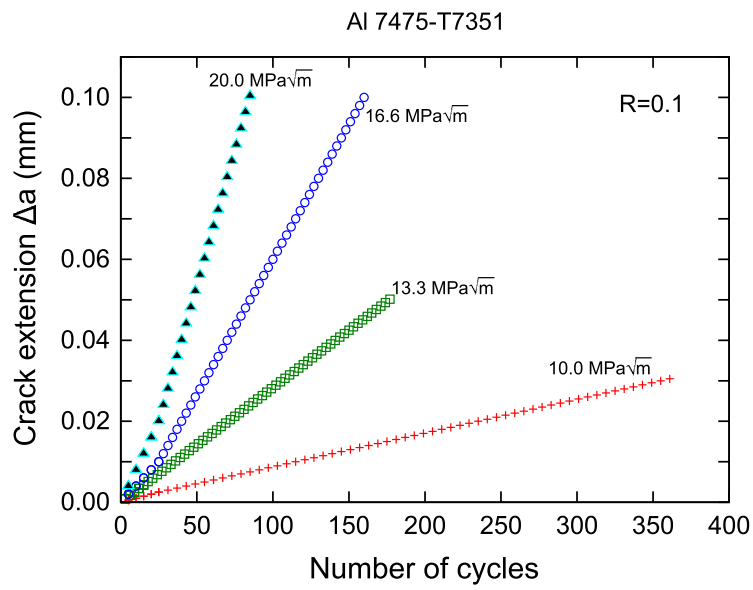

Fig. 9. Crack extension as a function of the number of cycles obtained as an output from the simulation.

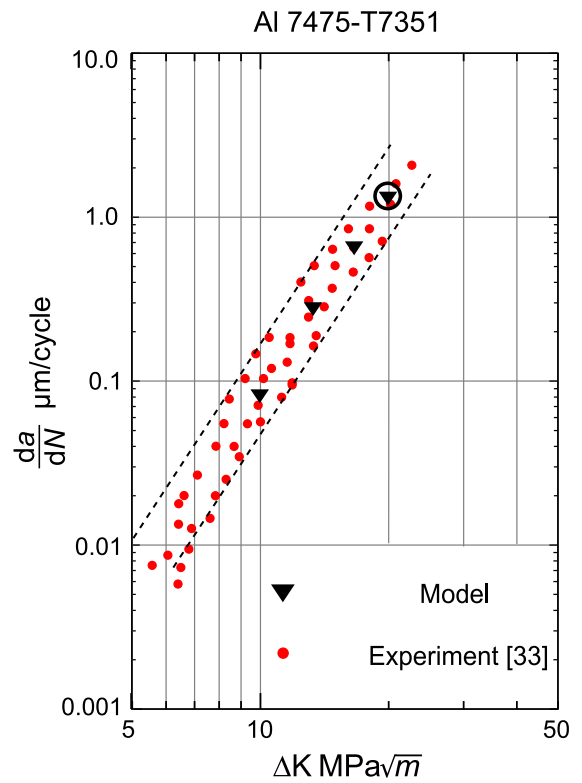

Fig. 10. Fatigue crack growth rates for Al 7475-T7351. Experimental data obtained from Ref. [33].

A power law fit of crack growth rate versus $\Delta K$ experimental data and that obtained from FE simulation using the current numerical scheme is shown in Fig. 12. As evident in the plot, a good match between the computed $d a / d N$ values and experimental results is obtained. Table 2 summarizes $W_{c r}^{p}$ and the Paris coefficients, $C$ and $m$ (Eq. (1)) obtained from the power law fits to experimental data and numerical results. The predicted values of $m$ are within $4 \%$ of the measured values and the predictions of $C$ are of the same order of magnitude as the measured data. 

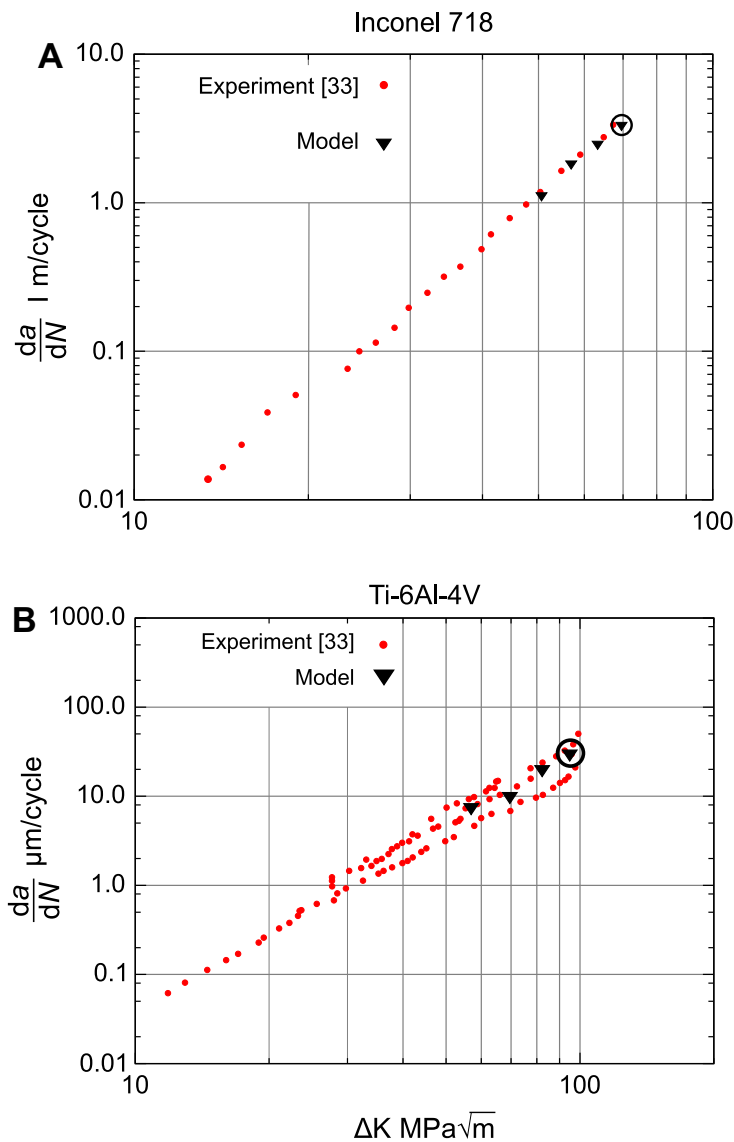

Fig. 11. Crack growth results for (A) Inconel 718 and (B) titanium alloy Ti-6AL-4V.

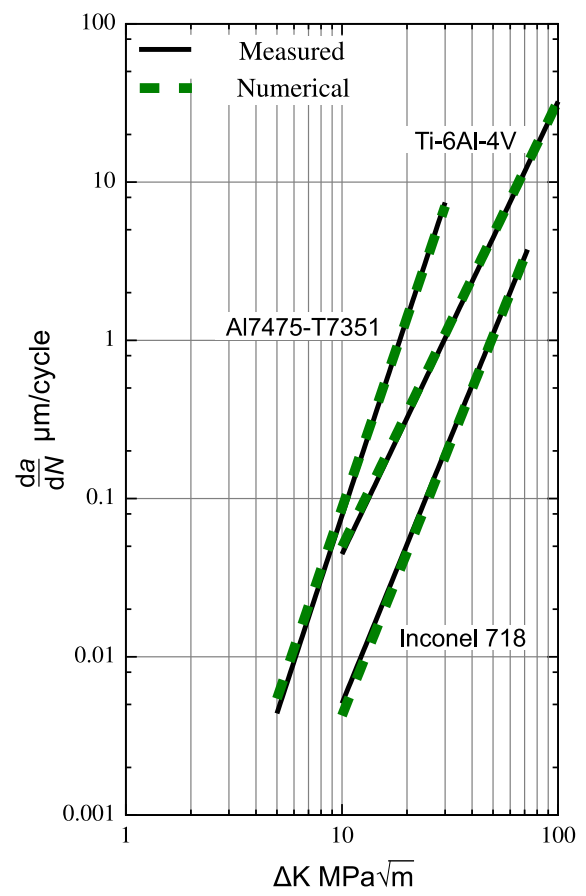

Fig. 12. Power law fit to measured data [33] and predictions from the proposed numerical model for selected metals. 
Table 2

Paris constants obtained from numerical simulation and measured data [33].

\begin{tabular}{|c|c|c|c|c|c|c|}
\hline & \multirow[t]{2}{*}{$W_{c r}^{p}\left(\mathrm{Nmm} / \mathrm{mm}^{2}\right)$} & \multicolumn{2}{|c|}{ Paris coefficient $(C) \times 10^{-06}$} & \multicolumn{3}{|c|}{ Paris exponent $(m)$} \\
\hline & & Measured & Model & Measured & Model & Relative error (\%) \\
\hline Al 7475-T7351 & 30 & 5.51 & 8.70 & 4.15 & 4.00 & 3.6 \\
\hline Inconel 718 & 86 & 2.32 & 1.58 & 3.34 & 3.43 & 2.7 \\
\hline $\mathrm{Ti}-6 \mathrm{Al}-4 \mathrm{~V}$ & 100 & 61.5 & 75.0 & 2.86 & 2.82 & 1.4 \\
\hline
\end{tabular}

\section{Concluding remarks}

This work presents a numerical scheme adopting the finite element method and a plastically dissipated energy criterion for simulating fatigue crack propagation. The propagation criterion is based on a condition that relates the cumulative increment in plastically dissipated energy ahead of the crack tip to a critical value. The accumulated increment in plastically dissipated energy is probed in a dissipation domain that fully encloses the reverse plastic zone ahead of the crack tip and the crack propagates when the criterion is fulfilled. Thus, the crack propagation rate in this growing crack model results from successive evaluation of the propagation criterion and not specified a priori.

Fatigue crack propagation was simulated in a standard compact specimen under 2D plane strain conditions. The above scheme for cyclic crack propagation was used to obtain the crack growth rate for a range of applied load levels, $\Delta K$. The critical plastically dissipated energy per unit crack extension was obtained from a single, experimentally measured $(\Delta K, d a / d N)$ data point. Once the critical value was established, crack growth for different levels of applied $\Delta K$ was modeled assuming a constant critical value. The resulting crack propagation rates were in good agreement with experimental data for three types of aerospace alloys. The numerically predicted values of the Paris exponent $m$ were within $5 \%$ of measured values. The reliability of the scheme is dependent on adequately resolving the reverse plastic zone with at least 4 elements.

The current numerical scheme is based on a growing crack model and thus shows promise for modeling variable amplitude loading conditions. A quantitative comparison with experimental data of crack growth under variable amplitude loading and enhancement of the scheme with cycle jump techniques for increasing the computational efficiency for such loading conditions are topics to be considered in future research. In all, this work confirms by comparing numerical simulations to experimental results that the critical dissipated energy can be used as a criterion to predict fatigue crack propagation.

\section{Acknowledgment}

The authors would like to gratefully acknowledge NSF for supporting this work through Grant CMMI-0825444.

\section{References}

[1] Dusan Krajcinovic. Damage mechanics, 41. Elsevier: North Holland; 1996.

[2] Lemaitre J, Desmorat R. Engineering damage mechanics: ductile, creep, fatigue and brittle failures. Springer Verlag; 2005.

[3] Paris PC, Gomez MP, Anderson WE. A rational analytic theory of fatigue. Trends Engng 1961;13:9-14.

[4] Elber W. Fatigue crack closure under cyclic tension. Engng Fract Mech 1970;2(1):37-45.

[5] Rice JR. Mechanics of crack tip deformation and extension by fatigue. Symposium on fatigue crack growth. ASTM-STP 1967;415:247-311.

[6] Bodner SR, Davidson DL, Lankford J. A description of fatigue crack growth in terms of plastic work. Engng Fract Mech 1983;17(2):189-91.

[7] Klingbeil NW. A total dissipated energy theory of fatigue crack growth in ductile solids. Int J Fatigue 2003;25(2):117-28.

[8] Cojocaru D, Karlsson AM. An object-oriented approach for modeling and simulation of crack growth in cyclically loaded structures. Adv Engng Software 2008;39(12):995-1009.

[9] Cojocaru D, Karlsson AM. Assessing plastically dissipated energy as a condition for fatigue crack growth. Int J Fatigue 2009;31(7):1154-62.

[10] Raju KN. An energy balance criterion for crack growth under fatigue loading from considerations of energy of plastic deformation. Int J Fract $1972 ; 8(1): 1-14$.

[11] Weertman J. Theory of fatigue crack growth based on a BCS crack theory with work hardening. Int J Fract 1973;9(2):125-31.

[12] Skelton RP, Vilhelmsen T, Webster GA. Energy criteria and cumulative damage during fatigue crack growth. Int J Fatigue 1998;20(9):641-9.

[13] Fine ME, Davidson DL. Quantitative measurement of energy associated with moving a fatigue crack, fatigue mechanisms: advances in quantitative measurement of physical damage. ASTM STP 1983;811:350-70.

[14] Ranganathan N, Petit J, de Fouquet J. Energy required for fatigue crack propagation. In: Proceedings of the 7th international conference on strength of metals and alloys, Montreal, Canada; 1986. p. 1267-72.

[15] Birol Y. What happens to the energy input during fatigue crack propagation? Mater Sci Eng: A 1988;104:117-24.

[16] Ranganathan N, Chalon F, Meo S. Some aspects of the energy based approach to fatigue crack propagation. Int J Fatigue 2008;30(10):1921-9.

[17] Smith KV. Application of the dissipated energy criterion to predict fatigue crack growth of type 304 stainless steel following a tensile overload. Engng Fract Mech 2011;78(18):3183-95.

[18] Dang-Van K. Macro-micro approach in high-cycle multiaxial fatigue. ASTM STP 1993;1191:120.

[19] Korsunsky AM, Dini D, Dunne FPE, Walsh MJ. Comparative assessment of dissipated energy and other fatigue criteria. Int J Fatigue 2007;29(911):1990-5.

[20] Laird C, Smith GC. Crack propagation in high stress fatigue. Philos Mag 1962;7(77):847-57.

[21] Daily JS, Klingbeil NW. Plastic dissipation in mixed-mode fatigue crack growth along plastically mismatched interfaces. Int J Fatigue 2006;28(12):1725-38.

[22] Nittur PG, Karlsson AM, Carlsson LA. Implementation of a plastically dissipated energy criterion for three dimensional modeling of fatigue crack growth. Int J Fatigue 2013:54:47-55. ISSN 0142-112.

[23] Anderson TL. Fracture mechanics: fundamentals and applications. CRC; 2005.

[24] Ellyin F. Fatigue damage, crack growth, and life prediction. Springer; 2001. 
[25] ABAQUS user's manual. Dassault Systémes. ABAQUS V10.2; 2010.

[26] Rossum G et al. Python programming language. URL <http://www.python.org $>$.

[27] ASTM E399-12. Standard test method for linear-elastic plane-strain fracture toughness $\mathrm{KI}_{c}$ of metallic materials. West Conshohocken (PA): ASTM International, 2012.

[28] Singh KD, Parry MR, Sinclair I. A short summary on finite element modelling of fatigue crack closure. J Mech Sci Technol 2011;25(12):3015-24.

[29] McClung RC, Sehitoglu H. On the finite element analysis of fatigue crack closure - 1. Basic modeling issues. Engng Fract Mech 1989;33(2):237-52.

[30] McClung RC. Finite element analysis of specimen geometry effects on fatigue crack closure. Fatigue Fract Engng Mater Struct 1994;17(8):861-72.

[31] Park SJ, Song JH. Simulation of fatigue crack closure behavior under variable-amplitude loading by a 2D finite element analysis based on the most appropriate mesh size concept. ASTM STP 1999;1343:337-50.

[32] Solanki K, Daniewicz SR, Newman JC. Finite element modeling of plasticity-induced crack closure with emphasis on geometry and mesh refinement effects. Engng Fract Mech 2003;70(12):1475-89.

[33] Military standardization handbook: metallic materials and elements for aerospace vehicle structures. Department of Defense; 2003.

[34] Davidson DL. The energetics of fracture. In: Morris E, editor. Fine symposium, TMS fall meeting, Detroit (MI); 1991. p. 355-62. 